\title{
Controlled Transportation of Light by Light at the Microscale
}

\author{
Manuel Crespo-Ballesteros $\odot^{*}$ and Misha Sumetsky $\odot$ \\ Aston Institute of Photonic Technologies, Aston University, Birmingham B4 7ET, United Kingdom
}

(Received 17 May 2020; accepted 16 March 2021; published 16 April 2021)

\begin{abstract}
We show how light can be controllably transported by light at microscale dimensions. We design a miniature device that consists of a short segment of an optical fiber coupled to transversally oriented inputoutput microfibers. A whispering gallery soliton is launched from the first microfiber into the fiber segment and slowly propagates along its $\mathrm{mm}$-scale length. The soliton loads and unloads optical pulses at designated input-output microfibers. The speed of the soliton and its propagation direction is controlled by the dramatically small, yet feasible to introduce, permanently or all-optically, nanoscale variations of the effective fiber radius.
\end{abstract}

DOI: 10.1103/PhysRevLett.126.153901

Transportation of objects by other objects, both at the macroscale and microscale, is an evident constituent of the evolution of nature in general and living beings in particular. At the macroscale, we travel and carry things from one place to another and use machines to make it easier and faster [1]. At the microscale, human-guided transportation and manipulation of objects is of great multidisciplinary importance, with applications ranging from medical and life science to nanomaterial science, bionanotechnology, and nanoelectronics [2].

In microphotonics, addressed in this Letter, we can separate the micro-objects under study into those constituted of matter (e.g., waveguides, microresonators, and micro or nanoparticles) and those constituted of light (e.g., optical waves, pulses, and localized states). Consequently, at the microscale we distinguish the transportation and manipulation of (a) matter by matter, (b) light by matter, (c) matter by light, and (d) light by light.

There are numerous examples of matter micro or nano-objects being controllably transported and manipulated by other matter micro or nano-objects. The developed approaches often resemble the manipulation of macroscopic objects with mechanical, electromechanical, and magnetic tools [2]. In particular, at the atomic-scale dimensions, the transportation and manipulation of atoms and nano-objects are possible with an atomic force microscope [3] [Fig. 1(a)].

Transportation of light by material micro-objects is possible as well. For example, microlasers and optical microresonators are used to confine and manipulate light at the microscale [4-6] [Fig. 1(b)]. They are commonly considered at rest with

Published by the American Physical Society under the terms of the Creative Commons Attribution 4.0 International license. Further distribution of this work must maintain attribution to the author(s) and the published article's title, journal citation, and DOI. respect to the laboratory system of reference [4,5,7]. Generally, the translation of a microresonator with constant speed does not affect the behavior of localized states residing in it [8]. However, accelerated translation, vibration, and rotation can significantly perturb the resonant states [9-13]. In the simplest case, light confined in a microresonator can be transported mechanically using a "truck" in the form of a translation stage. In another example, controlling the perturbation of an eigenstate in a rotating microresonator allows one to use it as a miniature gyroscope $[11,12]$.

In turn, light in the form of optical tweezers can confine and manipulate matter micro-objects [14,15]. For example, light waves can localize microparticles close to their antinodes by the gradient and scattering forces [Fig. 1(c)]. In addition, propagation of light through nonlinear media

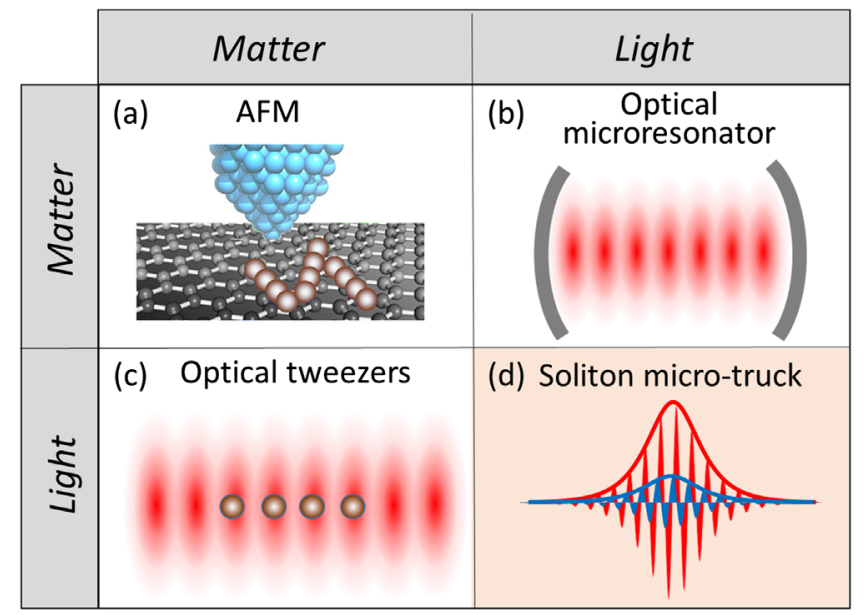

FIG. 1. Matter by matter, light by matter, matter by light, and light by light transportation at the microscale. (a) An atomic force microscope (AFM) tip translating atoms at the solid surface. (b) An optical microresonator translating an optical eigenstate. (c) An optical tweezers translating micro-objects. (d) An optical soliton translating an optical eigenstate. 
allows the manipulation of light itself, e.g., the modification of its spectrum and self-localization at the microscale. Examples of current significant interest include frequency comb generation [16,17], optomechanical processes [18], and the formation of solitons [17,19-21]. Conventionally, for telecommunication applications, broadband picosecond and subpicosecond temporal solitons are used $[19,20]$. Broadband solitons have been studied in application to frequency comb generation [16,17]. Slow broadband solitons with picosecond durations have been demonstrated in photonic crystals [22,23]. Slow narrowband solitons, which may have much a smaller propagation speed, can be realized in periodic microstructures provided that the soliton central frequency is close to the band gap edge [24-26].

However, can we use light as optical tweezers for light? Is it possible to confine and translate light controllably and all optically at the microscale? The natural approach to answer this question is to consider a soliton as a moving microresonator that can confine and transport weaker light and thus be used as a micro-truck for light [Fig. 1(d)]. This may be possible since the electric field $E$ of a soliton propagating along an optical fiber induces a change in the refractive index $\Delta n \sim|E|^{2}$ due to the nonlinear Kerr effect $[19,20]$. Therefore, the soliton field (as well as the field of any other sufficiently strong optical pulse) can act as a moving effective potential well that traps and transports an optical signal. This idea is conceptually different from the phenomena of nonlinear manipulation of light by light noted in the previous paragraph (e.g., formation, transformation, and interaction between solitons). In contrast, in the process described below, the soliton acts as an optical tweezers that (i) should not affect or be affected by the optical pulse it carries and (ii) is able to grab and release the carried pulse at request. Three decades ago, transportation of a localized optical state by an optical soliton was proposed $[27,28]$. This beautiful idea did not attract much attention because a realistic device that enables the all-optical transportation of a relatively weak state of light, including its loading and unloading, had not been suggested to date.

In this Letter, we describe a microdevice where relatively weak optical pulses and eigenstates are transported between input and output ports by a soliton (Fig. 2). The device consists of an uncoated optical fiber segment (FS) coupled to three transverse input-output microfibers: $\mathrm{MF}_{0}, \mathrm{MF}_{1}$, and $\mathrm{MF}_{2}$. In our model, a whispering gallery soliton (WGS) is formed by the resonant excitation of a whispering gallery mode launched by the $\mathrm{MF}_{0}$ inside the FS. The WGS slowly propagates along the surface of the fiber and further slows down near $\mathrm{MF}_{1}$, where a relatively weak whispering gallery pulse or eigenstate (WGE) is loaded. Similar to the slow linear propagation of whispering gallery modes realized in surface nanoscale axial photonics (SNAP) technology [29-32], we engineer the nanoscale variation of the fiber effective radius (corresponding to the sub-GHz variation of the cutoff frequency) so that the soliton can

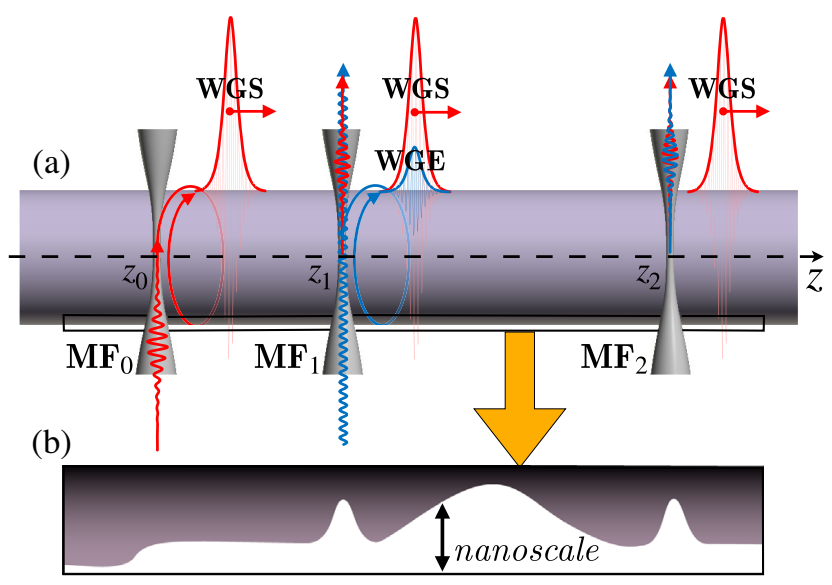

FIG. 2. Illustration of the conveyance of a weak optical pulse by a soliton. $\mathrm{MF}_{0}, \mathrm{MF}_{1}$, and $\mathrm{MF}_{2}$ are the input-output microfiber waists of biconical tapers coupled to the optical fiber segment. WGS is a whispering gallery soliton, and WGE is a relatively weak whispering gallery pulse or eigenstate transported by the WGS. $\mathrm{MF}_{0}$ serves as the WGS source, and $\mathrm{MF}_{1}$ and $\mathrm{MF}_{2}$ serve as stops where the WGE is loaded and unloaded.

slow down and stop, as well as reverse its propagation direction. In particular, the WGS can continue its propagation to $\mathrm{MF}_{2}$ where the WGE is unloaded, or turn back before reaching $\mathrm{MF}_{2}$ and unload the WGE back into $\mathrm{MF}_{1}$. Furthermore, we suggest that the required dramatically small variation of the fiber parameters along its length can be introduced all-optically.

A pulse with central angular frequency $\omega_{s}$ is coupled into the FS from $\mathrm{MF}_{0}$ forming a whispering gallery mode, which is enhanced due to the constructive self-interference. As a result, a WGS with central frequency $\omega_{s}$ is formed. It is assumed that $\omega_{s}$ is close to the cutoff frequency $\omega_{s}^{\text {(cut) }}(z)$ of the FS, which is slowly varying along the FS axis $z$. Because of the proximity of $\omega_{s}$ and $\omega_{s}^{\text {(cut) }}(z)$, the axial speed of the created WGS is small and, for this reason, sensitive to a small variation of $\omega_{s}^{(\mathrm{cut})}(z)$. Similarly, the central frequency of a weak WGE, $\omega_{e}$, different from $\omega_{s}$, is close to a slowly and weakly varying cutoff frequency $\omega_{e}^{\text {(cut) }}(z)$. Because of the small cutoff frequency variations $\Delta \omega_{s, e}^{(\mathrm{cut})}(z)=\omega_{s, e}^{(\mathrm{cut})}(z)-\omega_{s, e}$ assumed here, the expression for slowly propagating whispering gallery modes can be factorized as $R_{m_{s, e} p_{s, e}}(r) e^{i m_{s, e} \varphi} \psi_{s, e}(z, t) e^{-i \omega_{s, e} t}$ where $m_{s, e}$ and $p_{s, e}$ are azimuthal and radial quantum numbers. Consequently, the propagation of a narrow bandwidth WGS with central frequency $\omega_{s}$ and a WGE with frequency $\omega_{e} \neq \omega_{s}$ along the fiber axis coordinate $z$ is determined by their amplitudes $\psi_{s}(z)$ and $\psi_{e}(z)$. These functions are defined by a system of coupled nonlinear Schrödinger equations, which are similar to those commonly used in nonlinear fiber optics $[19,27,28]$ where the temporal and spatial coordinates are interchanged [24,25,33,34]. Assuming $\left|\psi_{s}(z)\right| \gg\left|\psi_{e}(z)\right|$, we have 


$$
\begin{gathered}
i \partial_{t} \psi_{s}=-\frac{1}{2} \kappa_{s} \partial_{z}^{2} \psi_{s}+\left[\Delta \omega_{s}^{(\mathrm{cut})}(z)+i \gamma_{s}+\kappa_{s} \sum_{j=0}^{2} D_{s j} \delta\left(z-z_{j}\right)-\frac{\omega_{s} n_{2}}{n_{s} A_{s s}}\left|\psi_{s}\right|^{2}\right] \psi_{s}+G_{s}(t) \delta\left(z-z_{0}\right), \\
i \partial_{t} \psi_{e}=-\frac{1}{2} \kappa_{e} \partial_{z}^{2} \psi_{e}+\left[\Delta \omega_{e}^{(\mathrm{cut})}(z)+i \gamma_{e}+\kappa_{e} \sum_{j=1}^{2} D_{e j} \delta\left(z-z_{j}\right)-2 \frac{\omega_{e} n_{2}}{n_{e} A_{s e}}\left|\psi_{s}\right|^{2}\right] \psi_{e}+G_{e}(t) \delta\left(z-z_{1}\right) .
\end{gathered}
$$

Here $\kappa_{s, e}=c^{2} /\left(n_{s, e}^{2} \omega_{s, e}\right), c$ is the speed of light, $n_{s}$ and $n_{e}$ are the refractive indices of the FS at frequencies $\omega_{s}$ and $\omega_{e}, n_{2}$ is its nonlinear refractive index, $\delta(z)$ is the delta function, and $A_{s s}, A_{s e}$ are the effective mode areas defined in [19] and the Supplemental Material [35]. The terms $G_{s}(t) \delta\left(z-z_{0}\right)$ and $G_{e}(t) \delta\left(z-z_{1}\right)$ in Eqs. (1a) and (1b) determine the soliton and weak pulse sources at microfibers, which are specified below. Parameters $D_{s j}$ and $D_{e j}$ are the coupling of the FS to microfibers at frequencies $\omega_{s}$ and $\omega_{e}$ determined in Ref. [31]. For a single input-output microfiber with a source, Eq. (1a) coincides with that obtained previously in [34].

To estimate the characteristic parameters of our device, we assume that the FS is uniform. Then Eqs. (1a) and (1b) can be solved analytically [19] yielding for WGS:

$\left|\psi_{s}^{(0)}(z, t)\right|^{2}=P_{s} \operatorname{sech}^{2}\left(\frac{z-v_{s} t}{L_{s}}\right), \quad P_{s}=\frac{c^{2} A_{s s}}{n_{s} n_{2} \omega_{s}^{2} L_{s}^{2}}$,

where $P_{s}$ is the soliton peak power, $L_{s}$ is the soliton characteristic width, and $v_{s}$ is the soliton velocity. After the substitution of $\left|\psi_{s}^{(0)}(z, t)\right|^{2}$ from Eq. (2), Eq. (1b) describes the propagation of the WGE along the FS with time-dependent cutoff frequency $\omega_{e}^{\text {(cut) }}(z)-\Delta \omega_{e}^{\max } \operatorname{sech}^{2}\left(z-v_{s} t / L_{s}\right)$, where

$$
\Delta \omega_{e}^{\max }=\frac{2 c^{2} \omega_{e} A_{s s}}{n_{e} n_{s} \omega_{s}^{2} L_{s}^{2} A_{s e}}=2 P_{s} \frac{\omega_{e} n_{2}}{n_{e} A_{s e}} .
$$

Equation (3) determines the maximum variation of the cutoff frequency caused by the WGS. Assuming that the WGE has the same speed as the WGS, we look for the solution of Eq. (1b) in the form $\psi_{e}^{(0)}(z, t)=\Phi(x) e^{i \alpha x+i \beta t}$, which depends on the dimensionless relative coordinate $x=z-v_{s} t / L_{s}$. Then $\Phi(x)$ satisfies the equation

$$
\frac{d^{2} \Phi(x)}{d x^{2}}+\left(\epsilon-\eta \operatorname{sech}^{2} x\right) \Phi(x)=0, \quad \eta=\frac{2 n_{e} \omega_{e}^{2} A_{s s}}{n_{s} \omega_{s}^{2} A_{s e}},
$$

where $\epsilon=L_{s}^{2} / c^{4}\left[2 n_{e}^{2} \omega_{e} c^{2}\left(\Delta \omega_{e}+\beta\right)-v_{s}^{2} n_{e}^{4} \omega_{e}^{2}\right]$. Parameter $\epsilon$ can be tuned by varying $\Delta \omega_{e}=\omega_{e}^{(c u t)}-\omega_{e}$. The eigenvalues $\epsilon_{m}$ of Eq. (4) corresponding to the localized states $\Phi_{m}(x)$ are

$$
\epsilon_{m}=-(\xi-m)^{2}, \quad \xi=\sqrt{\eta+1 / 4}-1 / 2,
$$

and the index $m=0,1, \ldots,[\xi]$. The integer part of $\xi$, $[\xi]$, is the total number of localized eigenstates that the WGS can support. If $\eta<2$, then Eq. (4) has a single eigenstate $\Phi_{0}(x)=\operatorname{sech}^{\xi} x$. For small values of $\eta$, we have $\xi \approx 2 \eta, \epsilon_{0} \approx 4 \eta^{2}$, and the characteristic width of this eigenstate is $x_{w}=1 / \eta$, which corresponds to the WGE axial width $z_{w}=L_{s} / \eta$.

Figure 3 shows three exemplary voyages of a WGE transported by a WGS along a silica FS with radius $r_{0}=20 \mu \mathrm{m}$ found by numerical solution of Eqs. (1a) and (1b) [35]. This figure includes three examples with the cutoff frequency variations shown in Figs. 3(a1)-3(a3), corresponding to the acceleration, slowing down, and stopping of WGS between $\mathrm{MF}_{1}$ and $\mathrm{MF}_{2}$. We design the FS profile to satisfy the condition of adiabaticity, which ensures that the shapes of WGS and WGE are not altered significantly during the propagation. In addition, the speed of the WGS near $\mathrm{MF}_{1}$ and $\mathrm{MF}_{2}$ is set to enable loading, safe transporting, and unloading of the WGE. The central frequency of the input source in Eq. (1a) generating the WGS at $\mathrm{MF}_{0}$ is set to $\omega_{s} / 2 \pi=225 \mathrm{THz}$. In order to arrive at the minimum possible WGS speed, this frequency is assumed to coincide with the value of the FS cutoff frequency at $\mathrm{MF}_{0}$. The characteristic width, speed, and duration of the created soliton is $L_{s} \sim 50 \mu \mathrm{m}$, $v_{s} \sim 0.5 \mathrm{~mm} / \mathrm{ns}$, and $T_{s} \sim 100 \mathrm{ps}$. The frequency of the plane wave entering the FS from $\mathrm{MF}_{1}$ and forming the WGE is set to $195 \mathrm{THz}$ plus a small shift to match one of the possible eigenfrequencies of the WGS-formed potential well. Attenuations of WGS and WGE are set to $\gamma_{s, e}=$ $3 \mathrm{MHz}$ (corresponding to a $Q$ factor $\sim 2 \times 10^{8}$ at frequency $\omega / 2 \pi=190 \mathrm{THz}$ [36,37]. Other parameters of our devices are described in the Supplemental Material [35]. The height of the potential well formed by the WGS found from Eq. (3) is $\Delta \omega_{e}^{\max } / 2 \pi \sim 2.6 \mathrm{GHz}$, while the WGS maximum power is $P_{s} \sim 70 \mathrm{~kW}$. From Eq. (5), this potential well can only support a single WGE. For the WGS to survive and in order to minimize the WGE perturbation during its loading and unloading, it is critical to minimize the WGS coupling to $\mathrm{MF}_{1}$ and $\mathrm{MF}_{2}$ while keeping the WGE coupling large. This can be achieved by appropriate phase matching the microfiber-WGE coupling at frequency $\omega_{e}$ and phase unmatching the microfiber-WGS coupling at frequency $\omega_{s}$ [38]. In our modeling, we set $D_{s 1}=D_{s 2}=0.005 i \mu \mathrm{m}^{-1}$ and $D_{e 1}=D_{e 2}=0.05 i \mu \mathrm{m}^{-1}$, where the latter correspond 

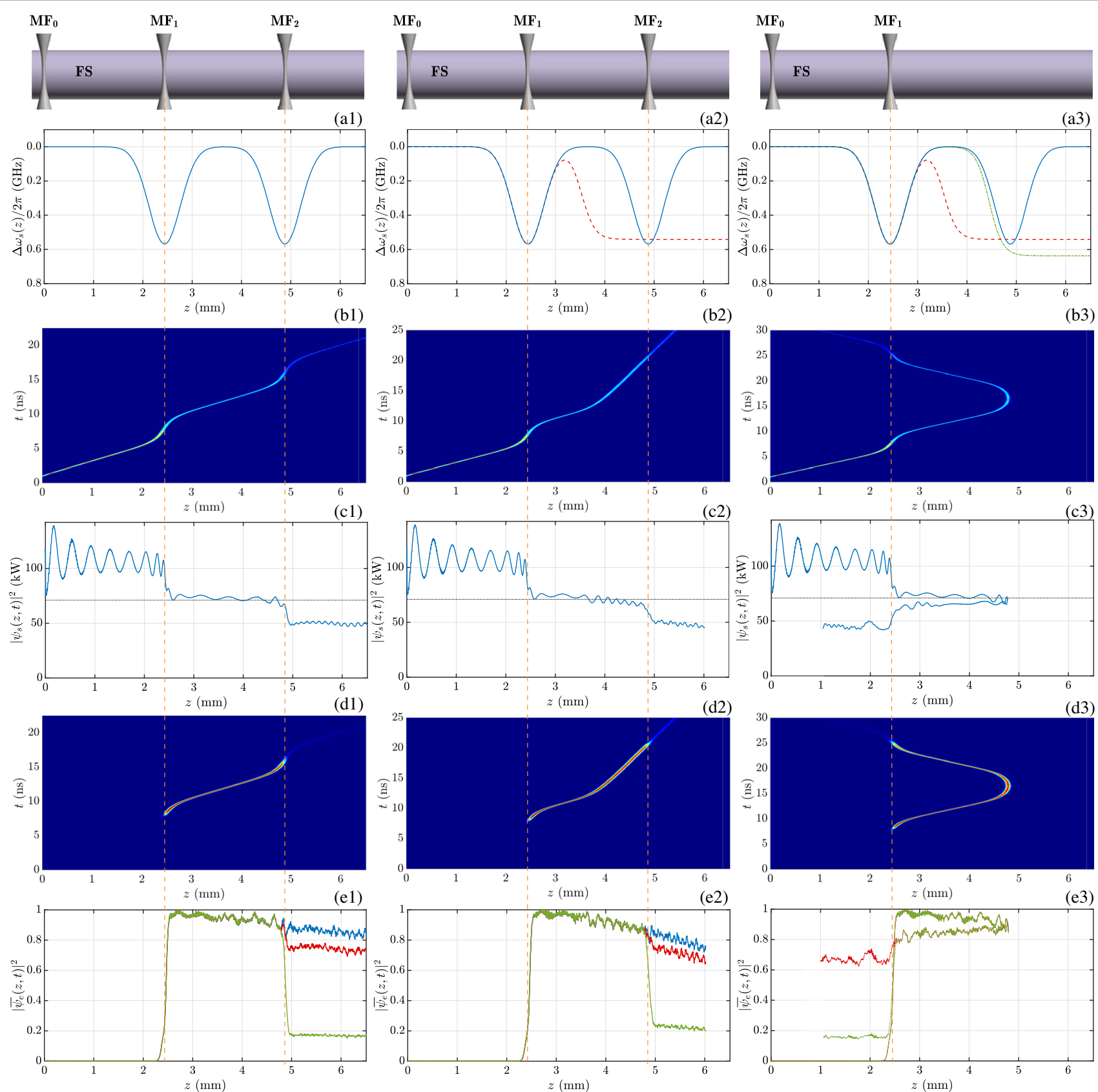

FIG. 3. Transportation of a WGE by a WGS. (a1)-(a3) Variation in the cutoff frequency introduced to control the speed of the WGS in the three cases considered (solid blue, dashed red, and dash-dotted green curves). (b1)-(b3) Evolution of WGS. (c1)-(c3) Variation of the WGS peak power as it propagates along the fiber. (d1)-(d3) Evolution of WGE positioned at the ground state of the WGS-induced quantum well. (e1),(e2) Variation of the normalized maximum of the WGE $\left|\bar{\psi}_{e}(z, t)\right|^{2}$ as it propagates along the fiber for different coupling coefficients with microfiber $\mathrm{MF}_{2}$ (red curves: $D_{s 2}=0.005 i \mu \mathrm{m}^{-1}$ and $D_{e 2}=0$; blue curves: $D_{s 2}=0$ and $D_{e 2}=0.05 \mu \mathrm{m}^{-1}$; green curves: $D_{s 2}=0.005 i \mu \mathrm{m}^{-1}$ and $D_{e 2}=0.05 i \mu \mathrm{m}^{-1}$ ). (e3) Same as (e1) and (e2) but only $\mathrm{MF}_{1}$ is present in this case.

to characteristic experimentally observed values [31,39]. We found that the real parts of these coupling parameters with the same order of magnitude do not noticeably modify the WGE [blue curve in Figs. 3(e1) and 3(e2)]. This is the reason why they are set to zero.

Figures 3(b1)-3(b3) show the propagation of the WGS considered in the examples. In Fig. 3(b1), the WGS speed in the intervals between input-output microfibers is $4.7 \times 10^{5} \mathrm{~m} / \mathrm{s}=0.0016 c$ and decreases to $0.9 \times$ $10^{5} \mathrm{~m} / \mathrm{s}=0.0003 \mathrm{c}$ near $\mathrm{MF}_{1}$ and $\mathrm{MF}_{2}$ for loading and unloading the WGE. Figure 3(b2)shows the propagation of the WGS when its speed is reduced to $1.3 \times 10^{5} \mathrm{~m} / \mathrm{s}=$ $0.00044 c$ in between microfibers. In Fig. 3(b3), only $\mathrm{MF}_{1}$ is present. The variation in the cutoff frequency introduced is such that the WGS stops at some distance ( $2.5 \mathrm{~mm}$ away from $\mathrm{MF}_{1}$ in the simulation) and returns back to $\mathrm{MF}_{1}$ to 
unload the WGE (see [29] for the analog dispersionless propagation of a linear pulse). Figures 3(c1)-3(c3) show the variation of the WGS power during its propagation. The simulations show the leakage of the WGS due to coupling with the microfibers. This effect is taken into account by the delta function in the fourth term of the right-hand side in Eq. (1a). In order not to destroy the WGS due to its leakage through $\mathrm{MF}_{1}$ and form an almost pure first order soliton ideal for transportation of signals between $\mathrm{MF}_{1}$ and $\mathrm{MF}_{2}$, the WGS original power was made high enough. Finally, Figs. 3(d1)-3(d3) and 3(e1)-3(e3) describe the loading, transport, and unloading of the WGE corresponding to the ground eigenstate of the WGS-formed quantum well. In order to load this eigenstate into the WGS-formed quantum well, we tuned the frequency of the input wave to match the frequency of this eigenstate. The detuning of the ground WGE eigenfrequency $\delta \omega / 2 \pi$ from the FS cutoff frequency is $0.88 \mathrm{GHz}$. Notice that a relatively small coupling $D_{s 2}$ still contributes to the WGE dissipation into the FS. The latter is found by setting $D_{e 2}=0$ [red curves in Figs. 3(e1)-3(e3)]. Taking this dissipation into account, we find that, for the $D_{e 2}=i 0.05 \mu \mathrm{m}^{-1}$ chosen, more than $70 \%$ of the WGE power is unloaded into $\mathrm{MF}_{2}$. Obviously, the amount of unloaded power can be improved by increasing $D_{e 2}$. Generally, the evolution of a WGS-trapped WGE pulse with frequencies distributed within the quantum well bandwidth (rather than coinciding with its eigenvalue) can be quite complex [40-42].

Thus, as in the case of slow linear propagation of whispering gallery modes [29-32], the speed of a slow WGS can be controlled by the dramatically small variation of the cutoff frequency along the optical fiber length. Such a WGS can serve as a moving optical microresonator-a soliton micro-truck - enabling programmed transportation of optical pulses and eigenstates, including their loading and unloading. Various other examples of transportation of a weak signal by solitons, as well as by optical pulses having power below the soliton formation threshold, can be considered. Of special interest is the investigation of nonadiabatic processes during the transportation of a WGE [43] and its loading and unloading. The characteristic peak power and duration of a WGS propagating along a silica fiber considered here are $100 \mathrm{~kW}$ and $100 \mathrm{ps}$, respectively. Such strong pulses may result in fiber damage ([35] and [44-46]). Therefore, further optimization of the carrier pulse parameters and speed may be required. However, according to our estimates [35], these pulses do not introduce a significant temperature variation. For chalcogenide and hydrogenated amorphous silicon (a-Si:H) fibers, which have a larger nonlinearity, the peak power of the WGS can be 2 orders of magnitude smaller $[47,48]$. As is well-known from quantum mechanics [49], the onedimensional potential well induced by a WGS can always hold at least one optical eigenstate despite its shallowness. One of the intriguing conclusions of our findings is that the
WGS speed can be fully controlled by an unexpectedly small variation of the cutoff wavelength $\Delta \omega_{s}^{\text {(cut) }} \sim 1 \mathrm{GHz}$, which, for the fiber radius $r_{0} \sim 20 \mu \mathrm{m}$, corresponds to an effective radius variation of $r_{0} \Delta \omega_{s}^{\text {(cut) }} / \omega_{s} \sim 0.1 \mathrm{~nm}$. The fabrication precision achievable in SNAP technologies $[29,32]$ makes the introduction of such dramatically small variations feasible. Furthermore, these variations can be induced all-optically. In fact, the amplitude of mechanical vibrations of an optical microresonator, which are induced by whispering gallery modes, can be tuned up to $10 \mathrm{~nm}$ [50]. For the microresonator with radius $r_{0} \sim 20 \mu \mathrm{m}$ considered in [50], this corresponds to a cutoff frequency variation exceeding $10 \mathrm{GHz}$. Launched through the same or additional control input-output microfibers, these modes can temporary induce the required variation of the cutoff wavelength. In this case, the behavior of the WGS (or a carrier pulse with power below the soliton threshold) and WGE are determined by the same Eq. (1) pair, where the cutoff frequency variations $\Delta \omega_{s, e}^{(\mathrm{cut})}(z, t)$ now depend on time $t$. Thus, the device described here potentially enables the all-optically controlled transportation of light by light at the microscale.

This research was funded by EPSRC Grant No. EP/ P006183/1. M. S. is grateful to A. A. Fotiadi and S. K. Turitsyn for useful discussions.

\section{* Corresponding author.} m.crespo@aston.ac.uk

[1] D. Teodorović and M. Janić, Transportation Engineering: Theory, Practice and Modeling (Elsevier, New York, 2016).

[2] Y. Sun and X. Liu, Micro- and Nano-Manipulation Tools (John Wiley \& Sons, New York, 2015); Z. Zhang, X. Wang, J. Liu, C. Dai, and Y. Sun, Robotic micromanipulation: Fundamentals and applications, Annu. Rev. Control Robotics Autonomous Syst. 2, 181 (2019); M. Makulavicius, O. Balitskyi, R. Urbonas, A. Dzedzickis, V. Bučinskas, A. Petronis, M. Kovalenko, I. Morkvenaite-Vilkonciene, Y. Sun, and X. Liu, Recent advances in mechanical micro- and nanomanipulation, in Automation 2020: Towards Industry of the Future. AUTOMATION 2020. Advances in Intelligent Systems and Computing, edited by R. Szewczyk, C. Zieliński, and M. Kaliczyńska (Springer, Cham, 2020), pp. 248-256, https://doi.org/10.1007/978-3-030-409715_23.

[3] G. Binnig, C.F. Quate, and C. Gerber, Atomic Force Microscope, Phys. Rev. Lett. 56, 930 (1986); B. Voigtländer, Atomic Force Microscopy, 2nd ed. (Springer International Publishing, New York, 2019); N. Santos and F. A. Carvalho, Atomic Force Microscopy, 1st ed. (Humana Press, Louisville, KY, 2019).

[4] K. J. Vahala, Optical microcavities, Nature (London) 424, 839 (2003).

[5] A. B. Matsko, Practical Applications of Microresonators in Optics and Photonics, 1st ed. (CRC Press, Boca Raton, FL, 2009). 
[6] F. Monticone and A. Alù, Metamaterial, plasmonic and nanophotonic devices, Rep. Prog. Phys. 80, 036401 (2017).

[7] M. R. Foreman, J. D. Swaim, and F. Vollmer, Whispering gallery mode sensors, Adv. Opt. Photonics 7, 168 (2015).

[8] Nevertheless, such translation can change the measured output signal as, e.g., in the case considered in this Letter when the resonator is moving with respect to the input-output waveguide. The output signal in this case depends on the relative speed of the resonator (Doppler effect).

[9] T. Carmon, H. Rokhsari, L. Yang, T. J. Kippenberg, and K. J. Vahala, Temporal Behavior of Radiation-PressureInduced Vibrations of an Optical Microcavity Phonon Mode, Phys. Rev. Lett. 94, 223902 (2005).

[10] A. A. Savchenkov, A. B. Matsko, V. S. Ilchenko, N. Yu, and L. Maleki, Whispering-gallery-mode resonators as frequency references. II. Stabilization, J. Opt. Soc. Am. B 24, 2988 (2007).

[11] W. Liang, V. S. Ilchenko, A. A. Savchenkov, E. Dale, D. Eliyahu, A. B. Matsko, and L. Maleki, Resonant microphotonic gyroscope, Optica 4, 114 (2017).

[12] Y.-H. Lai, Y.-K. Lu, M.-G. Suh, Z. Yuan, and K. Vahala, Observation of the exceptional-point-enhanced Sagnac effect, Nature (London) 576, 65 (2019).

[13] X. Jiang and L. Yang, Optothermal dynamics in whisperinggallery microresonators, Light Sci. Appl. 9, 24 (2020).

[14] A. Ashkin, J. M. Dziedzic, J. E. Bjorkholm, and S. Chu, Observation of a single-beam gradient force optical trap for dielectric particles, Opt. Lett. 11, 288 (1986).

[15] P. Zemánek, G. Volpe, A. Jonáš, and O. Brzobohatý, Perspective on light-induced transport of particles: From optical forces to phoretic motion, Adv. Opt. Photonics 11, 577 (2019).

[16] T. J. Kippenberg, R. Holzwarth, and S. A. Diddams, Microresonator-based optical frequency combs, Science 332, 555 (2011).

[17] T. J. Kippenberg, A. L. Gaeta, M. Lipson, and M. L. Gorodetsky, Dissipative Kerr solitons in optical microresonators, Science 361, eaan8083 (2018).

[18] M. Aspelmeyer, T. J. Kippenberg, and F. Marquardt, Cavity optomechanics, Rev. Mod. Phys. 86, 1391 (2014).

[19] G. Agrawal, Nonlinear Fiber Optics, 5th ed. (Academic Press, New York, 2012).

[20] Y. S. Kivshar and G. Agrawal, Optical Solitons: From Fibers to Photonic Crystals (Academic Press, New York, 2003).

[21] Y. V. Kartashov, B. A. Malomed, and L. Torner, Solitons in nonlinear lattices, Rev. Mod. Phys. 83, 247 (2011).

[22] P. Colman, C. Husko, S. Combrié, I. Sagnes, C. W. Wong, and A. De Rossi, Temporal solitons and pulse compression in photonic crystal waveguides, Nat. Photonics 4, 862 (2010).

[23] S. Malaguti, G. Bellanca, S. Combrié, A. de Rossi, and S. Trillo, Temporal Gap Solitons and All-Optical Control of Group Delay in Line-Defect Waveguides, Phys. Rev. Lett. 109, 163902 (2012).

[24] C. M. de Sterke and J. E. Sipe, Envelope-function approach for the electrodynamics of nonlinear periodic structures, Phys. Rev. A 38, 5149 (1988).
[25] N. A. R. Bhat and J. E. Sipe, Optical pulse propagation in nonlinear photonic crystals, Phys. Rev. E 64, 056604 (2001).

[26] J. T. Mok, C. M. de Sterke, I. C. M. Littler, and B. J. Eggleton, Dispersionless slow light using gap solitons, Nat. Phys. 2, 775 (2006).

[27] J. T. Manassah, Ultrafast solitary waves sustained through induced phase modulation by a copropagating pump, Opt. Lett. 15, 670 (1990).

[28] K. Steiglitz and D. Rand, Photon trapping and transfer with solitons, Phys. Rev. A 79, 021802(R) (2009).

[29] M. Sumetsky, Delay of Light in an Optical Bottle Resonator with Nanoscale Radius Variation: Dispersionless, Broadband, and Low Loss, Phys. Rev. Lett. 111, 163901 (2013).

[30] T. Hamidfar, K. V. Tokmakov, B. J. Mangan, R. S. Windeler, A. V. Dmitriev, D. L. P. Vitullo, P. Bianucci, and M. Sumetsky, Localization of light in an optical microcapillary induced by a droplet, Optica 5, 382 (2018).

[31] M. Sumetsky, Theory of SNAP devices: Basic equations and comparison with the experiment, Opt. Express 20, 22537 (2012).

[32] N. A. Toropov and M. Sumetsky, Permanent matching of coupled optical bottle resonators with better than $0.16 \mathrm{GHz}$ precision, Opt. Lett. 41, 2278 (2016).

[33] T. A. Weiss and Y. Sivan, Pulse propagation in the slow and stopped light regime, Opt. Express 26, 19294 (2018).

[34] S. V. Suchkov, M. Sumetsky, and A. A. Sukhorukov, Frequency comb generation in SNAP bottle resonators, Opt. Lett. 42, 2149 (2017).

[35] See Supplemental Material at http://link.aps.org/ supplemental/10.1103/PhysRevLett.126.153901 for details of the model and discussion of fiber losses and fiber damage thresholds.

[36] M. L. Gorodetsky, A. A. Savchenkov, and V. S. Ilchenko, Ultimate Q of optical microsphere resonators, Opt. Lett. 21, 453 (1996).

[37] M. Pöllinger, D. O'Shea, F. Warken, and A. Rauschenbeutel, Ultrahigh-Q Tunable Whispering-Gallery-Mode Microresonator, Phys. Rev. Lett. 103, 053901 (2009).

[38] B. E. Little, J.-P. Laine, and H. A. Haus, Analytic theory of coupling from tapered fibers and half-blocks into microsphere resonators, J. Lightwave Technol. 17, 704 (1999).

[39] D. L. P. Vitullo, S. Zaki, D. E. Jones, M. Sumetsky, and M. Brodsky, Coupling between waveguides and microresonators: The local approach, Opt. Express 28, 25908 (2020).

[40] E. Schrödinger, Der stetige Übergang von der Mikro- zur Makromechanik, Naturwissenschaften 14, 664 (1926); Translated into English in E. Schrödinger, Collected Papers in Wave Mechanics (Blackie \& Son, London, 1928), p. 41.

[41] M. Sumetsky, Microscopic optical buffering in a harmonic potential, Sci. Rep. 5, 18569 (2016).

[42] R. W. Robinett, Quantum wave packet revivals, Phys. Rep. 392, 1 (2004).

[43] S. Miyashita, Conveyance of quantum particles by a moving potential well, J. Phys. Soc. Jpn. 76, 104003 (2007).

[44] B. C. Stuart, M. D. Feit, A. M. Rubenchik, B. W. Shore, and M. D. Perry, Laser-Induced Damage in Dielectrics with Nanosecond to Subpicosecond Pulses, Phys. Rev. Lett. 74, 2248 (1995). 
[45] A. V. Smith, B. T. Do, G. R. Hadley, and R. L. Farrow, Optical damage limits to pulse energy from fibers, IEEE J. Sel. Topics Quantum Electron. 15, 153 (2009).

[46] N. Kuzuu, K. Yoshida, H. Yoshida, T. Kamimura, and N. Kamisugi, Laser-induced bulk damage in various types of vitreous silica at 1064, 532, 355, and $266 \mathrm{~nm}$ : Evidence of different damage mechanisms between 266-nm and longer wavelengths, Appl. Opt. 38, 2510 (1999).

[47] V.S. Shiryaev and M. F. Churbanov, Recent advances in preparation of high-purity chalcogenide glasses for mid-IR photonics, J. Non-Cryst. Solids 475, 1 (2017).
[48] N. Vukovic, N. Healy, F. H. Suhailin, P. Mehta, T. D. Day, J. V. Badding, and A. C. Peacock, Ultrafast optical control using the Kerr nonlinearity in hydrogenated amorphous silicon microcylindrical resonators, Sci. Rep. 3, 2885 (2013).

[49] L. D. Landau and E. M. Lifshitz, Quantum Mechanics: Non-Relativistic Theory, 3rd ed. (Butterworth-Heinemann, Oxford, 1981).

[50] G. Anetsberger, O. Arcizet, Q. P. Unterreithmeier, R. Rivière, A. Schliesser, E. M. Weig, J.P. Kotthaus, and T. J. Kippenberg, Near-field cavity optomechanics with nanomechanical oscillators, Nat. Phys. 5, 909 (2009). 